\title{
Isolated and Collective Magnetic Resonances in Dielectric Nanoparticles at Optical Frequencies
}

\author{
Shi-Qiang Li, Wuzhou Song and Kenneth B. Crozier \\ Department of Electrical and Electronic Engineering and School of Physics, University of Melbourne, VIC 3010 Australia \\ Authore-mail addresses: shiqiang.li@unimelb.edu.au,kenneth.crozier@unimelb.edu.au
}

\begin{abstract}
We employ multipole expansion to analyze radiation modification and light scattering by isolated nanoparticles and by nanoparticle ensembles, unveiling directional emission mediated by magnetic resonance and two types of purely magnetic resonances at optical frequencies.

OCIS codes: (310.6628) Subwavelength structure; (140.4780) Optical resonators.
\end{abstract}

\section{Introduction}

The magnetic dipole is the lowest order magnetic mode of the multipole expansion. It is currently holds much interest, being a critical building block of the Huygens metasurface. [1] Previous works have often used multipole expansion via integration over an imaginary sphere surrounding the scattering particle and/or the radiating dipole, but this faces challenges if there are multiple scattering particles and/or radiating dipoles. The discrete-dipole approximation has been used to find multipole coefficients in these cases but is only for scattering problems. [2] Here, we employ a novel method [3] to analyze the multipole coefficients of each scatterer (silicon nanorod) in an array and/or on a substrate, based on its internal field distribution. This allows us to show the role that magnetic dipole modes can play in directional emission, and to analyze two types of purely magnetic resonances.

\section{Results and Discussions}

We begin by considering directional emission for the case of an electric dipole sitting in the center of a 2dimensional square array of silicon nanorods (Fig. 1(a)). We choose the dipole polarization to be along the z-axis, so that the dipole radiation is mainly parallel to the plane of the array and subsequently the interaction between the array and the dipole is strong. The electric field generated on the surfaces of the nanoparticles is given in Fig. 1(a), showing that the interaction is strongest with the nearest four nanorods and decays rapidly with outward distance. The far field radiation patterns occurring for the dipole situated in arrays with different numbers of nanorods are plotted in Fig. 1(b). In the absence of nanorods, the radiation pattern is largely in-plane, with no radiation into the zdirection (Fig. 1(b.i)). However, even with the addition of just four nanorods, the radiation is strongly modified and directed out-of-plane (Fig.1(b.ii)). With additional nanorods, the directionality increases further (Fig.1(b.iii-b.v)).

We next decompose the scattering of an individual nanorod of the array into multipoles (Fig. 1(c)). It can be seen that the electric and magnetic dipoles (ED, MD) dominate and have similar strength, with negligible contribution from higher order multipoles, such as the electric and magnetic quadrupoles (EQ, MQ). In Fig.1(d), we plot the radiation pattern produced when the nanorods (64-nanorod case in Fig. 1(b.v)) are removed and replaced by electric and magnetic dipoles, whose strengths are found from the above multipole decomposition. It can be seen that this radiation pattern matches well to that of the real array (i.e. with the nanorods, Fig. 1(b.v)), implying that it is sufficient to represent the nanorods with equivalent electric and magnetic dipoles. Furthermore, we plot the separate contributions from $\mathrm{ED}$ and $\mathrm{MD}$, revealing that the directionality mainly results from the induced MD component. This reconfirms the importance of induced magnetic dipoles for directional emission.

We next use multipole expansion to examine two types of purely magnetic resonances. The first is a diffractively-coupled lattice resonance. Lattice resonances show promising properties for directional emission and lasing [4]. They have been generally based on arrays of electric dipoles, and the possibilities available with magnetic dipoles have not been explored much. In Fig. 2(a), we show for nanorods in a square lattice with appropriate period, very sharp double peaks occur at wavelengths slightly longer than the period. Note that these peaks are not from individual nanorod resonances, which are about 30 times weaker (Fig. 2(c)). Multipole decomposition reveals that the two peaks are induced electric and magnetic dipole resonances (Fig. 2(a)). Furthermore, as the induced dipoles result from the interaction with two orthogonal lattice resonances, they can be separated by lifting the degeneracy of the lattice resonances. By modifying the lattice spacing so that it is no longer square, the ED resonance is shifted and the peaks are fully resolved (Fig. 2(b)). This also increases the sharpness of the resonances; with the MD resonance having a full-width-at-half-maximum (FWHM) of $3 \mathrm{~nm}$ and a quality factor $>200$.

The second purely magnetic resonance we consider is that of an individual nanorod sitting on a perfect electrical conductor (PEC). Nanophotonic devices frequently employ metal films that can be approximated as PECs, 
e.g. gold at infrared wavelengths. It is therefore of practical importance to consider how nanorod resonances interact with PECs. Our physical interpretation of what to expect is as follows. Normal incidence illumination of a particle on a substrate should induce dipoles in the particle, oriented in the plane of the substrate. Image theory predicts that the interaction of the induced dipoles with the PEC surface can be understood by replacing the PEC with image dipoles. Each image dipole is located at the same distance below the PEC surface as the original induced dipole is above it. With this principle in mind, in the upper half of Fig. 2(b) we schematically illustrate magnetic dipoles supported by various Si nanoparticle configurations: a nanosphere, a nanorod, and a nanorod above a PEC. The simulated vectorial electric field patterns are shown in the lower half of Fig. 2(b). For the nanorod above the PEC, the origin of the coordinate system of the multipole modes is shifted down from the nanorod center to the PEC surface, as this is the center of symmetry due to the presence of the image dipoles. The dipole modes will then have modified multipole coefficients. In Fig. 2(c), we plot the contributions to the scattering cross section from the ED and MD components for full nanorod in vacuum and for a half nanorod on a PEC. As before, these are found via multipolar decomposition from the internal field distributions. We also plot the total scattering cross sections, found by integration of the fields over imaginary (reference) surfaces that surround the nanorods. It can be seen that there is excellent agreement between each total scattering cross section and the sum of the contributions from the ED and MD components, confirming the accuracy of the model. It can also be seen that the ED is quenched by the PEC.

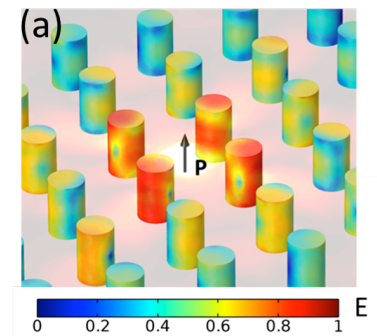

(b)

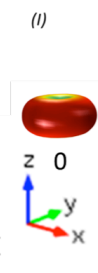

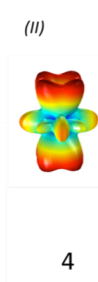

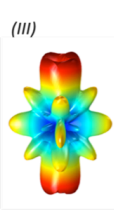

16

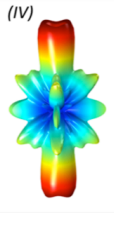

36 (c)

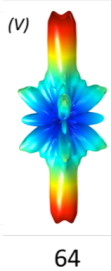

(d)

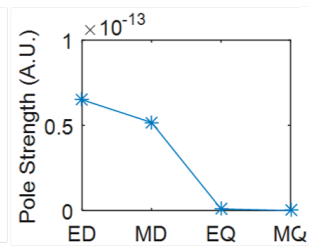

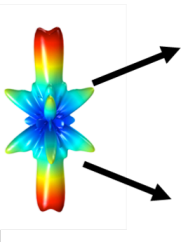

ED

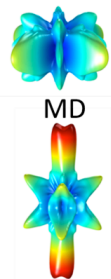

Fig. 1. (a) Perspective view of the radiation problem considered. The black arrow at the center is the radiating dipole and the surrounding pillars are the nanorods forming a square array. (b). The radiation patterns of a dipole with (i) no nanorods; (ii) 4; (iii) 16; (iv) 36; (v) 64; nanorods forming square lattices around the dipole. (c) The strengths of different orders of poles. (d) Left - radiation pattern from array of EDs and MDs. Right: Radiation patterns from arrays of EDs only (top) and MDs only (bottom). The scales are the same for all plots. Nanorods have radii of 70 $\mathrm{nm}$ and heights of $190 \mathrm{~nm}$. Period $=300 \mathrm{~nm}$. The principal axes of the arrays are aligned with $\mathrm{x}$ and $\mathrm{y}-$ axis.
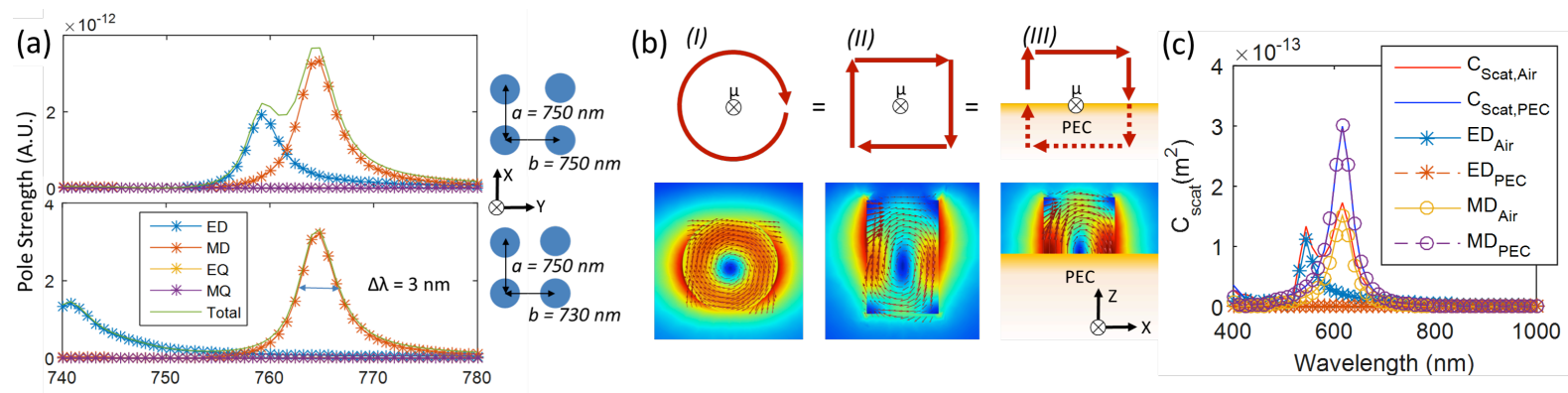

Fig. 2. (a) Strengths of ED and MD components of nanorod in arrays, with periods as indicated. (b) Side views of magnetic resonances induced by light incident normally on (I) a Si sphere (radius $=100 \mathrm{~nm}$ ) (II) Si nanorod (70 nm radius, $190 \mathrm{~nm}$ height) (III) Si nanorod (70 nm radius, 95 nm height) on PEC (c) The ED and MD components calculated for (b.II) and (b.III) and their scattering cross-sections calculated from simulation. In all cases of this Figure, the array or the particle is illuminated by light incident normally to the surface. The light polarization is along x-axis.

In summary, we use multipole expansion to study radiation and scattering from various dielectric nanoparticle configurations. Our work reveals that directional emission of a dipole in a dielectric nanorod array is mainly due to induced magnetic resonances. We furthermore introduced two types of purely magnetic resonances. Potential applications include ultra-high sensitivity optical sensors, flat optics, directional light sources, and tunable notch filters. The proposed structures should be readily achievable by modern micro- \& nano-fabrication techniques.

\section{References}

[1] M. Decker et al, "High efficiency dielectric Huygens' surfaces," Adv. Opt. Mat. 3, 813 (2015)

[2] Andrey B. Evlyukhin et al, "Optical response features of Si-nanoparticle arrays", Phys. Rev. B 82, 045404 (2010)

[3] Patrick Grahn et al, "Electromagnetic multipole theory for optical nanomaterials", New J. Phys. 14, 093033 (2012)

[4] W. Zhou et al, "Lasing action in strongly coupled plasmonic nanocavity arrays," Nature Nanotech. 8, 506-511 (2013). 